\title{
Fast matrix inversion methods based on Chebyshev and Newton iterations for zero forcing precoding in massive MIMO systems
}

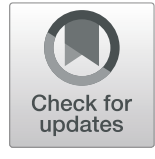

Sherief Hashima ${ }^{1 *}$ and Osamu Muta ${ }^{2}$

\begin{abstract}
In massive MIMO (mMIMO) systems, large matrix inversion is a challenging problem due to the huge volume of users and antennas. Neumann series (NS) and successive over relaxation (SOR) are two typical methods that solve such a problem in linear precoding. NS expands the inverse of a matrix into a series of matrix vector multiplications, while SOR deals with the same problem as a system of linear equations and iteratively solves it. However, the required complexities for both methods are still high. In this paper, four new joint methods are presented to achieve faster convergence and lower complexity in matrix inversion to determine linear precoding weights for mMIMO systems, where both Chebyshev iteration (Chebl) and Newton iteration ( $\mathrm{NI}$ ) are investigated separately to speed up the convergence of NS and SOR. Firstly, joint Chebyshev and NS method (Chebl-NS) is proposed not only to accelerate the convergence in NS but also to achieve more accurate inversion. Secondly, new SOR-based approximate matrix inversion (SOR-AMI) is proposed to achieve a direct simplified matrix inversion with similar convergence characteristics to the conventional SOR. Finally, two improved SOR-AMI methods, NI-SOR-AMI and Chebl-SOR-AMI, are investigated for further convergence acceleration, where $\mathrm{NI}$ and Chebl approaches are combined with the SOR-AMI, respectively. These four proposed inversion methods provide near optimal bit error rate (BER) performance of zero forcing (ZF) case under uncorrelated and correlated mMIMO channel conditions. Simulation results verify that the proposed Chebl-NS has the highest convergence rate compared to the conventional NS with similar complexity. Similarly, Chebl-SOR-AMI and NI-SOR-AMI achieve faster convergence than the conventional SOR method. The order of the proposed methods according to the convergence speed are Chebl-SOR-AMI, NI-SOR-AMI, SOR-AMI, then Chebl-NS, respectively. Chebl-NS has a low convergence because NS has lower convergence than SOR. Although Chebl-SOR-AMI has the fastest convergence rate, $\mathrm{NI}$-SOR-AMI is preferable than ChebI-SOR-AMI due to its lower complexity and close inversion result.
\end{abstract}

Keywords: Massive MIMO, Matrix inversion, Neumann series, Successive over relaxation, Chebyshev iteration, Newton iteration

\section{Introduction}

Massive MIMO (mMIMO) is one of the most promising technologies for the 5th generation (5G) communication systems [1]. mMIMO recent applications include machine type communications, drone communications, control circuits in nuclear reactors, and nuclear physics applications. Its channel hardening property ensures mitigating the effect of noise and interference as the number

*Correspondence: eng_shrif@yahoo.com

$\dagger$ Equal contributor

${ }^{1}$ Engineering Dept, Egyptian Atomic Energy Authority, 13759 Inshas, Egypt

Full list of author information is available at the end of the article of antennas increase [2]. Hence, linear precoding methods can approximately achieve optimal performance in mMIMO systems [3]. However, there are challenging problems in practical implementation of mMIMO systems such as large matrix inversion resulted from the large number of users and antennas.

Large matrix inversion is an important practical issue that affects the precoder design and performance. A good precoder depends on matrix inversion approximation characteristics such as low complexity and good approximation accuracy. Generally, precoding methods are divided into linear and non-linear ones. Non-linear precoding methods such as constant enveloper (CE), dirty 
paper coding (DPC) [4], vector perturbation (VP), lattice aided, and Tomlinson-Harashima precoding (THP) are unfriendly to hardware implementation due to their high complexity [5]. Hence, linear precoders such as matched filter (MF), zero forcing (ZF), regularized zero forcing (RZF), phased ZF (PZF), and minimum mean square error (MMSE) are favorable although they need the inversion of channel matrix containing all users [6]. Direct, iterative, and expansions methods are three main categories to calculate large matrix inverse for linear precoding. Direct methods suffer from high complexity as it depends mainly on transferring the matrix to be inverted into a multiplication of simple matrices like QR and Chelosky decomposition [7]. Iterative methods belong to the family of solving linear equations such as Richardson method [8], conjugate gradient (CG) method [9], successive over relaxation (SOR) [10], symmetric successive over relaxation (SSOR) [11], and Gauss-Seidel (GS) method [12]. They have acceptable performance in mMIMO systems. However, these approaches provide indirect matrix inversion approximation as they calculate a product containing matrix inversion and quadrature amplitude modulation (QAM) symbol vectors. In addition, matrix inversion is required separately for specific calculations such as sum rate computations and rapid matrix modifications $[13,21]$. The matrix inverse can be directly updated (column added and column deleted) to save the matrix inversion time and complexity. Hence, these methods require more complexity for these specific calculations as the symbol vector is divided. Chebyshev iteration (ChebI) and Newton iteration (NI) provide fast convergence characteristics while their complexity depends on the number of iterations involved $[14,15]$. However, both iterative methods require complex calculation of initial input to ensure convergence. The third category, expansion methods, transfers the inverse of a matrix into a series of matrix vector products like Neumann series (NS) [16]. Although NS has slow convergence rate, it not only can approximate matrix inversion separately but also owns simple hardware implementation property $[17,18]$. In [19], the authors utilize NI to achieve faster convergence than ordinary NS. This inspired us to replace the quadrature order NI with cubic order ChebI to achieve not only more accurate inversion results but also faster convergence. NS and SOR are recent two research directions to reduce the complexity of matrix inversion in linear precoding. However, their convergence speed should be improved. This motivates us to speed up their convergence using cubic order ChebI.

Although the large precoding gain can be obtained by making use of large number of antennas, interference become dominant factor rather than additive noise. Hence, under these circumstances, ZF precoder is a reasonable choice compared to other precoding methods. So our focus in this paper is on ZF precoding technique for mMIMO systems to reduce its complexity. In this paper, four new joint methods are proposed to achieve faster convergence with reasonable complexity in matrix inversion to determine linear precoding weights for mMIMO systems, where the first iteration result of Chebyshev iteration (ChebI) and Newton iteration (NI) approaches are employed to reconstruct both NS and SOR methods. A high probability of convergence is achieved that can offer useful guidelines for practical mMIMO systems. The main contributions of this paper are five folds.

- Firstly, we propose a new joint Chebyshev iteration and Neumann series (ChebI-NS) method that not only achieves faster convergence but also provides more accurate matrix inversion approximation than previous NS methods.

- Secondly, we propose a new SOR-based approximate matrix inversion (SOR-AMI) method that directly approximates matrix inversion by separating the QAM symbol vector from the whole iteration process. The new method, which is very useful for further calculations, achieves the same convergence rate as SOR method with lower complexity.

- Thirdly, to further improve convergence characteristics of SOR-AMI, we propose joint NI and SOR-AMI method (NI-SOR-AMI), where we adopt one NI iteration to get an efficient searching direction for the following SOR-AMI iterations to achieve a fast convergence rate.

- Fourthly, another method to accelerate the convergence of SOR-AMI is to make use of cubic order ChebI instead of quadrature order NI. Hence, joint ChebI and SOR-AMI method (ChebI-SOR-AMI) is the fourth proposed technique that achieves faster convergence rate.

- Finally, the above four proposed methods are compared with existing methods in order to prove their faster convergence with reasonable near-ideal ZF 1 performance of downlink (DL) mMIMO system. Based on these results, we discuss the effectiveness of the proposed approaches.

The rest of this paper is organized as follows. Section 2 discusses the system model, mMIMO channel model, and preliminaries about NS expansion, SOR, proposed SORAMI method, NI, and Chebyshev iterations. Section 3 describes the four new proposed joint matrix inversion methods. Section 4 presents computational complexity analysis. Simulation results are introduced in Section 5. Finally, Section 6 concludes the work.

${ }^{1}$ In ideal ZF, exact value of Gram matrix is used. Thus, the result corresponds to case where Gram matrix inverse is obtained with sufficient accuracy. 
Notations: Upper-case and lower-case boldface letters denote matrices and vectors, respectively. $(.)^{T},(.)^{H},(.)^{-1}$, $(.)^{(n)}$, and $(.)^{\dagger}$ present transpose, conjugate transpose (Hermitian), inversion, $n^{\text {th }}$ iteration number, and pseudo inverse, respectively. $C \sim N\left(\mu, \sigma^{2} \mathbf{I}_{K}\right)$ denotes the circularly symmetric complex Gaussian distribution with mean $\mu$ and co-variance matrix $\sigma^{2} \mathbf{I}_{K}$ where $\mathbf{I}_{K}$ is the identity matrix of size $K$. $\|$.$\| and \|.\|_{2}$ define the 1-norm and 2-norm, respectively.

\section{System model and preliminaries}

In this section, we will carefully describe our system model followed by mMIMO channel model. Also, related matrix inversion approaches such as NS, SOR, NI , and ChebI are briefly introduced.

\subsection{System model}

Figure 1 shows a DL centralized mMIMO system with $N$ antennas equipped at the base station (BS) and serves $K$ $<<N$ single antenna users [1]. If the DL transmitted signal vector after precoding is $\mathbf{x} \in C^{N \times 1}$, the received signal vector $\mathbf{y} \in C^{K \times 1}$ for $K$ users can be expressed as:

$$
\mathbf{y}=\sqrt{\omega} \mathbf{H x}+\mathbf{n},
$$

where $\mathbf{n} \in C^{K \times 1}$ is the additive white Gaussian noise vector with zero mean and unit variance. $\omega$ is a normalization factor to determine signal to noise power ratio (SNR), i.e., SNR is given as $S N R=\frac{\omega}{\sigma^{2}=1}=\omega$, where $\sigma^{2}$ denotes additive noise variance. $\mathbf{H} \in C^{K \times N}$ is the DL channel matrix ${ }^{2}$. Furthermore, $\mathbf{H}=\left[\mathbf{h}_{1}, \mathbf{h}_{2}, \ldots ., \mathbf{h}_{k}\right]^{T}$, where $\mathbf{h}_{k} \in C^{1 \times N}$ is the channel vector between the BS and the $k^{\text {th }}$ user modeled as an independent and identically distributed (i.i.d) random vector. $\mathbf{x}$ is precoded using the ZF precoder and defined as:

$$
\mathbf{x}=\mathbf{P s}=\beta \mathbf{H}^{\dagger} \mathbf{s}=\beta \mathbf{H}^{H}\left(\mathbf{H} \mathbf{H}^{H}\right)^{-1} \mathbf{s}=\beta \mathbf{H}^{H} \mathbf{W}^{-1} \mathbf{s},
$$

where $\mathbf{s} \in C^{K \times 1}$ is the symbol vector of 64 QAM symbols from $K$ users for transmission [1], $\mathbf{P} \in C^{N \times K}$ is the ZF precoding matrix, $\mathbf{W} \in C^{K \times K}$ is the Gram matrix defined as $\mathbf{H H}^{H}$, and $\beta$ is a normalization parameter defined as $\sqrt{\frac{K}{t r\left(\mathbf{W}^{-1}\right)}}[22]$, where $\operatorname{tr}\left(\mathbf{W}^{-1}\right)$ defines the trace of $\mathbf{W}^{-1}$. In this paper, we assume perfect channel state information (CSI) at the BS by utilizing the time domain training pilot [23]. In time division duplex (TDD) mMIMO systems, the BS uses the user pilots to estimate the uplink channel. Hence, the DL CSI is achieved using channel reciprocity property in TDD systems.

\footnotetext{
${ }^{2}$ Two channel models are considered in the next subsection: (i) uncorrelated Rayleigh channel, named $\mathbf{H}_{u n} \in C^{K \times N}$, with Gaussian distribution of zero mean and unit variance, and (ii) spatial correlated channel, named $\mathbf{H}_{c o} \in C^{K \times N}$, as in [23].
}

It is obvious from (2) that the main complexity for ZF precoding is the inversion of $K \times K$ matrix $\mathbf{W}$. The Gram matrix $\mathbf{W}$ is Hermitian positive definite as in Eq. (3).

$$
\mathbf{u}^{H} \mathbf{W} \mathbf{u}=\mathbf{u}^{H}\left(\mathbf{H H}^{H}\right) \mathbf{u}=\mathbf{u}^{H} \mathbf{H}\left(\mathbf{u}^{H} \mathbf{H}\right)^{H}
$$

where $\mathbf{u}$ is an arbitrary $K \times 1$ non-zero vector.

The columns of the channel matrix $\mathbf{H}$ are asymptotically orthogonal and thus $\mathbf{H}$ is a full rank matrix [1]. $\mathbf{u H}$ equals zero vector only when $\mathbf{u}$ is a zero vector. Hence, we have $\mathbf{u H}(\mathbf{u H})^{H}>0$ for all non-zero vectors indicating that $\mathbf{W}$ is a positive definite matrix.

\section{2 mMIMO channel model}

This paper considers not only uncorrelated Rayleigh channel but also spatially correlated ones. The elements of uncorrelated channel, $\mathbf{H}_{u n} \in C^{K \times N}$, are independent and identically distributed (i.i.d.) complex Gaussian random variables (RVs) with zero mean and unit variance. On the other hand in the spatially correlated MIMO channel $\mathbf{H}_{c o}$ [24], the Kronecker channel model [25], in which $\mathbf{H}_{c o} \in$ $C^{K \times N}$, can be modeled as:

$$
\mathbf{H}_{c o}=\mathbf{R}_{r x}^{\frac{1}{2}} \mathbf{H}_{u n} \mathbf{R}_{t x}^{\frac{1}{2}}
$$

where $\mathbf{R}_{r x} \in C^{K \times K}$ and $\mathbf{R}_{t x} \in C^{N \times N}$ are the correlation matrices for the receive and transmit antennas, respectively. Since we assume single antenna user, then $\mathbf{R}_{r x}=\mathbf{I}$ [26]. Note that if also $\mathbf{R}_{t x}$ equals identity matrix, the left hand side of Eq. (4) will be the uncorrelated channel $\mathbf{H}_{u n}$. The (p, q) element of exponentially correlated transmit correlation matrix $\mathbf{R}_{t x}$ is given as [25]:

$$
R_{t x}(p, q)=\left(\zeta e^{j \Psi}\right)^{q-p},
$$

where $0 \leq \zeta \leq 1$ denotes the correlation magnitude between adjacent transmit antennas and $\Psi$ is the phase.

\subsection{Neumann series (NS) method}

According to the Neumann series expansion [17], the required Gram matrix to be inverted, $\mathbf{W} \in C^{K \times K}$, is approximated as a sum of matrix polynomials.

$$
\mathbf{W}^{-1}=\sum_{n=0}^{+\infty}\left(\mathbf{I}_{K}-\boldsymbol{\phi} \mathbf{W}\right)^{n} \boldsymbol{\phi},
$$

where $\phi \in C^{K \times K}$ preconditioning matrix and $\mathbf{I}_{K}$ is the $K \times K$ identity matrix. Assumptions of $\phi$ and the proposed approach to determine it are given in Section 3.1.

The main condition of Eq. (6) to be fulfilled is

$$
\lim _{n \rightarrow \infty}\left(\mathbf{I}_{K}-\boldsymbol{\phi} \mathbf{W}\right)^{n}=\mathbf{0}_{K}
$$




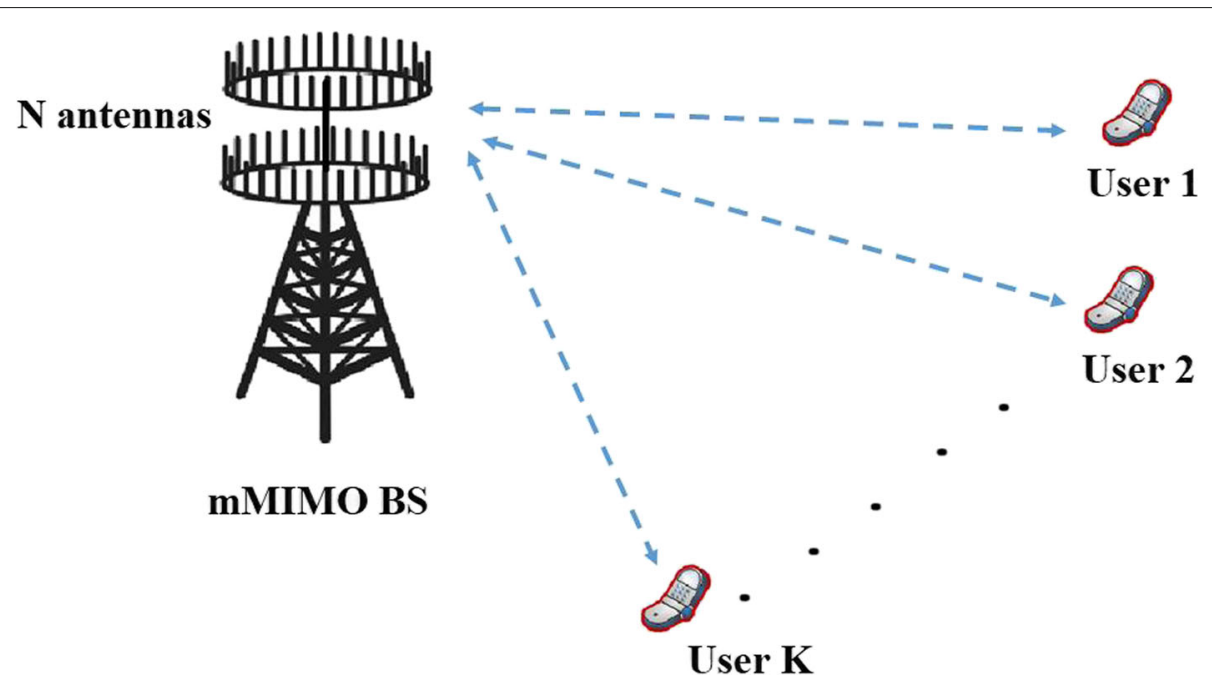

Fig. 1 Massive MIMO system model in downlink, where $K$ and $N$ denote number of single antenna users and number of antennas per BS, respectively. This figure describes the utilized system model which is one BS with $N$ antennas that serve $K$ single antenna users

where $\mathbf{0}_{K}$ is a zero matrix of size $K \times K$. For practical use, the inverse, $\mathbf{W}^{-1}$, is approximated according to the value of $L$ which is the maximum number of iterations ${ }^{3}$

$$
\mathbf{W}^{-1} \approx \hat{\mathbf{W}}^{-1}=\sum_{n=0}^{L}\left(\mathbf{I}_{K}-\boldsymbol{\phi} \mathbf{W}\right)^{n} \boldsymbol{\phi},
$$

where $L$ is the iteration number and $\hat{\mathbf{W}}^{-1}$ is the approximated inverse.

\subsection{SOR method}

The SOR method aims to iteratively solve the Gram matrix inversion problem as a linear equation $\mathbf{W g}=\mathbf{s}$, where $\mathbf{g}$ is an unknown vector solution of length $K \times 1$. The matrix $\mathbf{W}$ is decomposed into

$$
\mathbf{W}=\mathbf{D}+\mathbf{L}+\mathbf{U} \text {, }
$$

where $\mathbf{D}, \mathbf{L}$, and $\mathbf{U}=\mathbf{L}^{H}$ are the diagonal component, lower triangular component, and upper triangular component of Hermitian positive definite matrix $\mathbf{W}$.

If $\mathbf{W g}=\mathbf{s}$, i.e., $\mathbf{g}=\mathbf{W}^{-1} \mathbf{s}$, the $n^{\text {th }}$ estimation of $\mathbf{W}^{-1} \mathbf{s}$ is obtained by substituting Eq. (9) into the SOR method equation as follows [10]:

$$
\mathbf{g}^{(n)}=(\mathbf{D}+\alpha \mathbf{L})^{-1}\left(\alpha \mathbf{s}+((1-\alpha) \mathbf{D}-\alpha \mathbf{U}) \mathbf{g}^{(n-1)}\right),
$$

where $n$ defines the number of iterations, $\mathbf{g}^{(n)}$ is the $n^{\text {th }}$ iteration of $\mathbf{g}$ which also equals the SOR $n^{\text {th }}$ estimation of $\mathbf{W}^{-1} \mathbf{s}$, and $\alpha$ is the relaxation parameter. The utilized

\footnotetext{
${ }^{3}$ Mainly, $L$ is the number of expanded terms. However, in this paper, $L$ equals the number of iterations(i) so as to compare NS convergence with other methods. The maximum value of $L=4$, i.e., 4 th iteration as it provides a good trade-off between complexity and performance [20].
}

optimal relaxation parameter in this paper according to [10] equals

$$
\alpha_{\text {opt }}=0.404 e^{\left(-0.323 \frac{N}{K}\right)}+1.035 .
$$

Note that SOR method computes a product that contains the matrix inverse, i.e., $\mathbf{g}^{(n)}$ is the $n^{\text {th }}$ estimation of $\mathbf{W}^{-1} \mathbf{s}$.

\subsection{Newton iteration (NI)}

Newton iteration method can be employed to calculate $\mathbf{W}^{-1}$ in an iterative way [14]. Assume that $\mathbf{Z}^{(0)}$ is the initial estimation inverse of $\mathbf{W}^{-1}$ and

$$
\left\|\mathbf{I}_{K}-\mathbf{W} \mathbf{Z}^{(0)}\right\|<1 \text {. }
$$

Hence, the $(n+1)^{t h}$ iteration estimation of $\mathbf{W}^{-1}$ using NI is obtained by substituting $f(\mathbf{Z})=\mathbf{Z}^{-1}-\mathbf{W}$ in NI function $\mathbf{Z}^{(n+1)}=\mathbf{Z}^{(n)}-\frac{f\left(\mathbf{Z}^{(n)}\right)}{f^{\prime}\left(\mathbf{Z}^{(n)}\right)}$, where $f^{\prime}()$ is first derivative function of a function whose argument is a matrix as defined in $[14,15]$. The final NI formula that calculates the $(n+1)^{t h}$ estimation of $\mathbf{W}^{-1}$ is expressed as $[14,15]$ :

$$
\mathbf{Z}^{(n+1)}=\mathbf{Z}^{(n)}\left(2 \mathbf{I}-\mathbf{W} \mathbf{Z}^{(n)}\right),
$$

where $n$ denotes the number of iterations. If $n$ is large, Eq. (13) is converged to the Gram matrix inverse, i.e., $\mathbf{W}^{-1}$.

\subsection{Chebyshev iteration (Chebl)}

Chebyshev iteration is a third order convergence algorithm [15]. Similar to NI, substitute the function $f(\mathbf{Z})=$ $\mathbf{Z}^{-1}-\mathbf{W}$ into Chebyshev three terms function $\mathbf{Z}^{(n+1)}=$ $\mathbf{Z}^{(n)}-\frac{f\left(\mathbf{Z}^{(n)}\right)}{f^{\prime}\left(\mathbf{Z}^{(n)}\right)}-\frac{f^{\prime \prime}\left(\mathbf{Z}^{(n)}\right)}{2 f^{\prime}\left(\mathbf{Z}^{(n)}\right)}\left(\frac{f\left(\mathbf{Z}^{(n)}\right)}{f^{\prime}\left(\mathbf{Z}^{(n)}\right)}\right)^{2}$ to get the matrix inversion using ChebI, where $f^{\prime \prime}()$ is the second derivative 
function. Note that the third term of Chebychev $\mathbf{Z}^{(n+1)}$ helps ChebI to provide more accurate results than NI. The $(n+1)^{\text {th }}$ Chebyshev iteration expression of $\mathbf{W}^{-1}$ is expressed as [15]:

$$
\mathbf{Z}^{(n+1)}=\mathbf{Z}^{(n)}\left(3 \mathbf{I}-\mathbf{W} \mathbf{Z}^{(n)}\left(3 \mathbf{I}-\mathbf{W} \mathbf{Z}^{(n)}\right)\right) .
$$

If number of iterations is sufficient, Eq. (14) is converged to the matrix inverse, i.e., $\mathbf{W}^{-1}$.

\section{Proposed methods}

In this section, we will discuss four proposed methods to speed up large matrix inversion calculation. We will start with the first proposal (i.e., ChebI-NS), and then, we will move to the second proposal (i.e., SOR-AMI). To achieve further improvement, we also propose improved SOR-AMI methods as the third and fourth proposal (i.e., ChebI-SORAMI and NI-SOR-AMI).

\subsection{Joint Chebyshev iteration and Neumann series method (Chebl-NS)}

The initial NS value, $\boldsymbol{\phi}$ in Eq. (6), greatly affects the convergence. The method of selecting $\phi$ plays an important role in NS acceleration. There are three assumptions to get $\boldsymbol{\phi}$ where two of them depend on the special properties of the matrices while the third one focus on getting the initial from other iterations like NS and ChebI. The popular assumption of $\boldsymbol{\phi}$ is the matrix inversion of $K \times K$ diagonal matrix $\mathbf{D}$ whose entries are the main diagonal elements of the Gram matrix $\mathbf{W}$, i.e., $\mathbf{D}^{-1}$ [17]. The matrix $\mathbf{D}^{-1}$ can be calculated as follows [18]:

$$
\mathbf{D}^{-1}=\operatorname{diag}\left(\frac{1}{w_{1,1}}, \ldots . ., \frac{1}{w_{k, k}}, \ldots . ., \frac{1}{w_{K, K}}\right),
$$

where $w_{k, k}$ is the $k^{t h}$ diagonal element of Gram matrix W.

The second assumption of $\boldsymbol{\phi}$ is $\left(\frac{\mathbf{I}_{K}}{N+K}\right)$ which also represents a diagonal matrix [1]. This is due to that the largest and smallest eigenvalues of Gram matrix $\mathbf{W}$ depends on $N$ and $K$. As the number of $N$ and $K$ grows, the eigenvalues of the gram matrix converges to a fixed distribution [17]. The third assumption is to utilize the first iteration output of NI, $\mathbf{Z}^{(1)}$ as in Eq. (13) with $n=0$ to initialize NS [19]. Initializing NS with ChebI instead of NI not only provides accurate inversion approximation but also speeds up NS convergence. The advantages of ChebI over NI such as fast convergence and more accurate approximation motivated us to initialize NS with the output of the first iteration of ChebI instead of NI.

In this paper, ChebI is applied first to provide a suitable $\boldsymbol{\phi}$ to speed up the convergence of NS. The joint ChebI-NS approach main steps to estimate $\mathbf{W}^{-1}$ are:

Step 1 Obtain the inverse of the diagonal matrix of Gram matrix W, i.e, $\mathbf{D}^{-1}$ as in Eq. (15).
Step 2 Apply one Chebyshev iteration (i.e., $\mathrm{n}=0$ in Eq.(14) with initial input $\mathbf{Z}^{(0)}=\mathbf{D}^{-1}$ as follows:

$$
\begin{aligned}
\mathbf{Z}^{(1)} & =\mathbf{Z}^{(0)}\left(3 \mathbf{I}-\mathbf{W} \mathbf{Z}^{(0)}\left(3 I-\mathbf{W} \mathbf{Z}^{(0)}\right)\right) \\
& =\mathbf{D}^{-1}\left(3 \mathbf{I}-\mathbf{W} \mathbf{D}^{-1}\left(3 \mathbf{I}-\mathbf{W} \mathbf{D}^{-1}\right)\right) .
\end{aligned}
$$

Step 3 Apply the obtained first ChebI, $\mathbf{Z}^{(1)}$, as an initial to the Neumann series as follows:

$$
\mathbf{W}^{-1} \approx \hat{\mathbf{W}}^{-1}=\sum_{n=0}^{L}\left(\mathbf{I}_{K}-\mathbf{Z}^{(1)} \mathbf{W}\right)^{n} \mathbf{Z}^{(1)}
$$

An approximated solution, $\hat{\mathbf{W}}^{-1}$, is obtained for finite number of iterations.

Lemma 1 For DL mMIMO systems, the Neumann series with initial value from Chebyshev iteration, $\boldsymbol{\phi}=\mathbf{Z}^{(1)}$, have a high probability convergence when [16]

$$
\eta \approx \frac{N}{K}>5.83
$$

Proof See Appendix A.

Equation (18) has a practical applications in mMIMO systems as it identifies the suitable number of BS antennas to the number of single antenna users in mMIMO systems. For example, $\eta=8$ and $\eta=16$ produce two typical downlink mMIMO configuration $N \times K=256 \times 32$ and $256 \times 16$ [12]. According to [16, 19], these values ensures high probability of convergence of 0.999 due to the large values of $\eta$.

\subsection{SOR-based approximate matrix inversion method (SOR-AMI)}

Our main idea is provided in the following Lemma

Lemma $2 \mathbf{W}^{-1}$ can be approximated to $\mathbf{R}^{(n)}$ when $n \rightarrow$ $\infty$ using iterative SOR method as follows:

$$
\mathbf{R}^{(n)}=(\mathbf{D}+\alpha \mathbf{L})^{-1}\left(\alpha \mathbf{I}_{K}+((1-\alpha) \mathbf{D}-\alpha \mathbf{U}) \mathbf{R}^{(n-1)}\right),
$$

where $\mathbf{R}^{(0)}$ is the initial input and chosen to be the diagonal component, i.e., $\mathbf{D}^{-1}, \mathbf{R}^{(n)}$ is the $n^{\text {th }}$ direct estimation of $\mathbf{W}^{-1}$. An approximated solution, $\hat{\mathbf{W}}^{-1}$, is obtained for finite number of iterations.

Proof See Appendix 6.

The SOR-AMI main steps, to directly estimate $\mathbf{W}^{-1}$, are as follow:

Step 1 Calculate the initial input $\mathbf{R}^{0}=\mathbf{D}^{-1}$ from Eq. (15).

Step 2 Apply the obtained $\mathbf{R}^{0}$ on the SOR-AMI method as in Eq.(19). 
Since $\mathbf{W}$ is Hermitian positive definite, the SOR method is convergent [10]. Hence, as $\mathbf{W}^{-1} \mathbf{s}$ is approximated by $\mathbf{g}^{(n)}(n \rightarrow \infty), \mathbf{W}^{-1}$ can be approximated by $R^{(n)}(n \rightarrow \infty)$. The SOR-AMI method based on Eq. (19) can be utilized to directly calculate $\mathbf{W}^{-1}$. According to Eq. (26), it has the same convergence of SOR iterative method.

\subsection{Improved SOR-AMI methods}

The convergence of SOR-AMI is accelerated by making use of the fast convergence property of NI and ChebI which is revealed at the beginning of the iteration for SORAMI method. Hence, ChebI-SOR-AMI and NI-SOR-AMI are discussed below, respectively.

\subsubsection{Joint Chebyshev iteration and SOR-AMI method (Chebl-SOR-AMI)}

The joint algorithm main procedures, to directly estimate $\mathbf{W}^{-1}$ using ChebI-SOR-AMI, are as follows:

Step 1 Apply one Chebyshev iteration with initial input $\mathbf{Z}^{(0)}=\mathbf{D}^{-1}$ as in Eq. (16).

Step 2 Use the obtained first ChebI, $\mathbf{Z}^{(1)}$, to apply on the SOR-AMI method as follows:

$\mathbf{Z}^{(n)}=(\mathbf{D}+\alpha \mathbf{L})^{-1}\left(\alpha \mathbf{I}_{K}+((1-\alpha) \mathbf{D}-\alpha \mathbf{U}) \mathbf{Z}^{(n-1)}\right), n \geq 2$.

Since $\mathbf{W}$ is Hermitian positive definite, the ChebI-SORAMI method is convergent as SOR-AMI has the same convergence of traditional SOR method. Equation (20) calculates $\mathbf{W}^{-1}$ after initializing SOR-AMI method with one iteration of ChebI, i.e., $\mathbf{Z}^{(n)} \approx \mathbf{W}^{-1}$. As the number of iterations approaches infinity, i.e., $(n \rightarrow \infty)$, Eq. (20) converges to the exact matrix inverse, i.e., $\mathbf{W}^{-1}$. An approximated solution, $\hat{\mathbf{W}}^{-1}$, is obtained by finite number of iterations.

\subsubsection{Joint Newton iteration and SOR-AMI (NI-SOR-AMI)}

Similar to ChebI-SOR-AMI method, NI-SOR-AMI depends on applying one NI as initial input to SORAMI. The main steps, to estimate $\mathbf{W}^{-1}$ directly using NI-SOR-AMI, are as follow:

Step 1 Apply one Newton iteration with initial input $\mathbf{Z}^{(0)}=\mathbf{D}^{-1}$ as follows:

$$
\mathbf{Z}^{(1)}=\mathbf{Z}^{(0)}\left(2 \mathbf{I}-\mathbf{W} \mathbf{Z}^{(0)}\right)=\mathbf{D}^{-1}\left(2 \mathbf{I}-\mathbf{W} \mathbf{D}^{-1}\right)
$$

Step 2 Apply the first NI, $\mathbf{Z}^{(1)}$, obtained from step 1 to SOR-AMI method similar to Eq. (20).

Similar to ChebI-SOR-AMI, NI-SOR-AMI is convergent and $\mathbf{W}^{-1}$ can be approximated by $\mathbf{Z}^{(n)}(n \rightarrow \infty)$ resulted from step 2. Similarly to the previous, an approximated solution, $\hat{\mathbf{W}}^{-1}$, is obtained for finite number of iterations. The main advantage of this method is its reduced complexity compared with ChebI-SOR-AMI method. Next section discusses the complexity analysis of the proposed methods.

\section{Complexity analysis}

In this paper, we evaluate the computational complexity analysis of the proposed methods in terms of required number of complex multiplications which is more popular and complicated. The channel coherence interval $T_{c}$, defined as the product of coherence time and coherence bandwidth, is under consideration for fair complexity comparison. There are two types of approaches that can solve (2). The first type that include our four proposed methods is to directly compute $\mathbf{W}^{-1}$ every channel coherence interval. Thus, $\mathbf{W}^{-1}$ can be calculated regardless of $T_{c}$, while it requires other auxiliary processing which increases the total complexity as $T_{c}$ increases. On the other hand, the other type is to calculate the precoding weight recursively as a product of $\mathbf{W}^{-1} \mathbf{s}$ such as SSOR method. Thus, overall complexity is increased as $T_{c}$ (i.e., the number of symbols per $T_{c}$ ) increases.

The complexity of ZF precoding within $T_{c}$ is $O\left(K^{3}+\right.$ $\left.T_{c} N K\right)$. The NS complexity for different initial $\phi$ values and more than two iterations (i.e., $n>2)$ is $O\left(K^{3}\right)$ compared to the exact matrix inversion. NS implements matrix multiplication and matrix addition which are favorable in hardware as no divisions are required $[1,18]$. From Eq. (14), one ChebI requires two matrix additions and three matrix multiplications. However, one NI complexity is reduced by one matrix addition and one matrix multiplications according to Eq.(13). When $\boldsymbol{\phi}=\mathbf{D}^{-1}$ or $\boldsymbol{\phi}=\frac{\mathbf{I}_{K}}{N+K}$, the complexity of Eq. (8) is $O\left(K^{2}\right)$ for the first iteration (i.e., $n=1$ ) and $O\left(K^{3}\right)$ for further iterations (i.e., $n \geq 2$ ). Note that for ChebI-NS, the complexity increases at $i=2$ due to the added multiplications because of applying one ChebI.

For the SOR-AMI method, the complexity is $\mathrm{O}\left(K^{2}+\right.$ $\left.T_{c} N K\right)$ as only two matrix multiplications are required . This means that SOR-AMI convergence is faster than NS and also has lower complexity especially at large iteration numbers. The computational complexity of NI-SOR-AMI is slightly lower than ChebI-SOR-AMI by one matrix multiplication and one matrix addition.

The overall complexities of the proposed methods in addition to NS, NI-NS [19], and SSOR [11] are shown in Table 1 considering the channel coherence interval $T_{c}$. Small $T_{c}$ values do not greatly affect our proposed methods complexity because it is defined as complexity per the number of symbols during $T_{c}$. Hence, it greatly reduces SSOR method complexity. For large $T_{c}$ values, if the product of $T_{c} N K$ is larger than $K^{3}$, then the complexity is increased with $T_{c}$ increment; otherwise, it has small effect compared to $K$ value. SOR-AMI complexity is lower than SSOR and in the same time it directly computes $\mathbf{W}^{-1}$. ChebI-NS complexity $O\left(K^{3}\right)$ is close to its 
Table 1 Complexity analysis ( $N$ number of BS antennas, $K$ number of users, $i$ iteration number, $T_{C}$ channel coherence interval)

\begin{tabular}{llll}
\hline Method & $i=2$ & $i=3$ & $i=4$ \\
\hline NS & $3 K^{2}-K+T_{c} N K$ & $K^{3}+K^{2}+T_{c} N K$ & $2 K^{3}+T_{c} N K$ \\
Chebl-NS & $3 K^{3}+2 K^{2}+T_{c} N K$ & $4 K^{3}+K^{2}+T_{c} N K$ & $5 K^{3}+T_{c} N K$ \\
NI-NS [19] & $2 K^{3}+2 K^{2}+T_{c} N K$ & $3 K^{3}+K^{2}+T_{c} N K$ & $4 K^{3}+T_{c} N K$ \\
SOR-AMI & $2 K^{2}+T_{c} N K$ & $3 K^{2}+T_{c} N K$ & $4 K^{2}+T_{c} N K$ \\
Chebl-SOR-AMI & $3 K^{3}+T_{c} N K+2 K^{2}$ & $3 K^{3}+T_{c} N K+3 K^{2}$ & $3 K^{3}+T_{c} N K+4 K^{2}$ \\
NI-SOR-AMI & $2 K^{3}+T_{c} N K+K^{2}$ & $2 K^{3}+T_{c} N K+2 K^{2}$ & $2 K^{3}+T_{c} N K+4 K^{2}$ \\
SSOR [11] & $T_{c}\left(6 K^{2}+3 K+N K\right)$ & $T_{c}\left(8 K^{2}+3 K+N K\right)$ & $T_{c}\left(10 K^{2}+3 K+N K\right)$ \\
\hline
\end{tabular}

traditional NS methods. Note that SSOR method estimates the matrix inversion using two SOR iterations in both the forward and reverse order. Also, SSOR calculates the matrix inverse indirectly, i.e., $\mathbf{W}^{-1} \mathbf{s}$, while other methods compute it directly that is highly recommended for fast matrix inverse updates [21]. The proposed SOR-AMI method has the lowest complexity compared to other methods. Initializing SOR-AMI with either NI or ChebI slightly increases its complexity but provides faster convergence results to achieve close inversion results at low iterations.

\section{Results and discussion}

To evaluate the effect of the proposed method, we conducted computer simulation. System model is the same as in Section 2.1. In the three proposed methods (i.e., ChebINS, ChebI-SOR-AMI, and NI-SOR-AMI), the initial values of Newton and Chebyshev iterations are the diagonal component of W. To evaluate the proposed methods, the Frobenius norm error and bit error rate (BER) as performance metrics. Un-coded system is assumed during simulation. Also, the average BER of all users are calculated during simulation. The MSE is defined as follows.

$$
F_{\text {error }}=\left\|\mathbf{W}^{-1}-\hat{\mathbf{W}}^{-1}\right\|_{F}
$$

where $\mathbf{W}^{-1}$ and $\hat{\mathbf{W}}^{-1}$ are defined as ideal inverse of the Gram matrix and approximated solution by the three abovementioned proposed methods. The ZF precoding with exact matrix inversion of $\mathbf{W}$ is added to our results as the benchmark. Two configurations $N \times K=256 \times 32$ and $N \times K=128 \times 16$ are considered. The utilized modulation scheme is 64 QAM. The parameters of correlated channel model are set to $\zeta=0.1$ and $\Psi=60^{\circ}$ phase shift.

Figure 2 shows the Monte Carlo simulation results for the Frobenius norm error between exact Gram matrix inverse and its approximated inverse against number of BS antennas, $N$, for NI-NS, ChebI-NS, SOR-AMI, NI-SORAMI, and ChebI-SOR-AMI methods under uncorrelated channel conditions after 10,000 MC trials and for second, third, and fourth iterations, respectively. The MSE is plotted against $N$ to measure the inversion error for each proposed scheme. Our error calculations neglects the modulation effect as our main focus is on the error resulted from precoding matrix inversion approximation. At the second iteration, The error of SOR-AMI method is the largest followed by NI-NS method that have the largest 2-norm error at the following two iterations. According to Lemma 1 , when $N$ is known, the number of users $K$ can be easily calculated and there will be a high convergence probability of the inversion. As small $N$ values, the error decreases because of the small matrix inversion dimensions. The figure illustrates the merits of initializing NS and SOR-AMI with ChebI. The three terms Chebyshev iteration is more accurate than NI in spite of increased computational complexity by one matrix addition and multiplication. Also, Because SOR-AMI convergence is faster than NS convergence, the MSE for the three based SOR-AMI methods are lower than ChebI-NS and NI-NS methods.

For ease of illustration and discussion, the next three figures divide the results into three parts. Figure $3 \mathrm{com}-$ pares the proposed ChebI-NS with other NS-based methods. Figure 4 do the same but for SOR-AMI, ChebI-SORAMI, NI-SOR-AMI with SOR based methods. Finally, Fig. 5 compares the all four proposed methods with each other. Figure 3a shows the BER against SNR of NI-NS, diagonal-based NS, new ChebI-NS, and NS with initial $\frac{I_{K}}{N+K}$ under uncorrelated channel conditions with $N \times K=$ $128 \times 16$ at the second iteration. Figure $3 \mathrm{~b}$ and $\mathrm{c}$ are for third and fourth iterations, respectively. From the three sub-figures, the new ChebI-NS algorithm has the superior performance close to ZF followed by the NI-NS method at the third and fourth iteration and has close performance to NI-NS at the second iteration. Figure $3 \mathrm{~d}, \mathrm{e}$, and $\mathrm{f}$ show the same analysis performed under correlated channel conditions with $\zeta=0.1$ and $60^{\circ}$ phase shift for the second, third, and fourth iteration, respectively. It is worth noting that at the second iteration, i.e., Fig. 3a, the NINS [19] converges slightly faster than ChebI-NS, but the reverse occurs under correlated channel conditions, i.e., Fig. 3c. Their performance is still not close to the optimal ZF. Hence, their matrix inversion results lack accuracy 


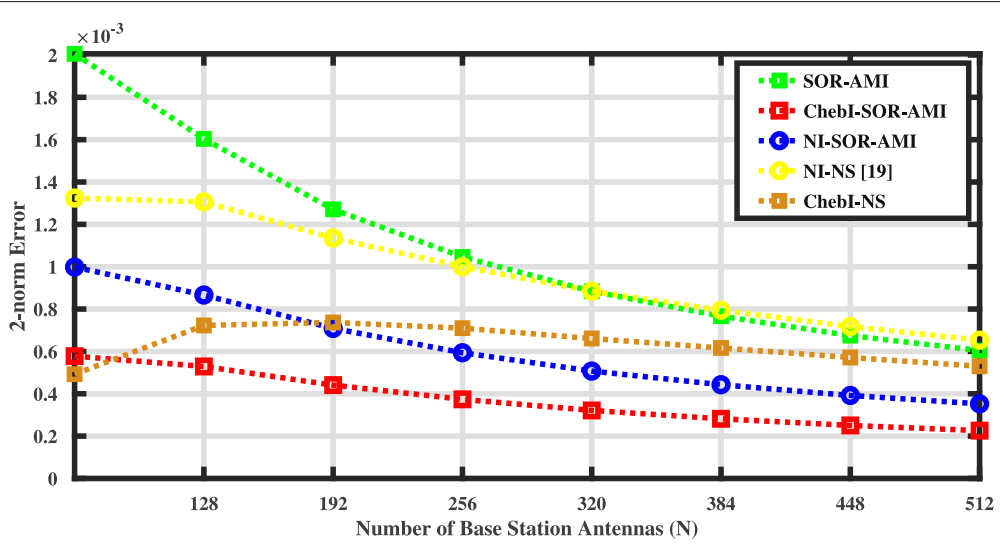

(a)

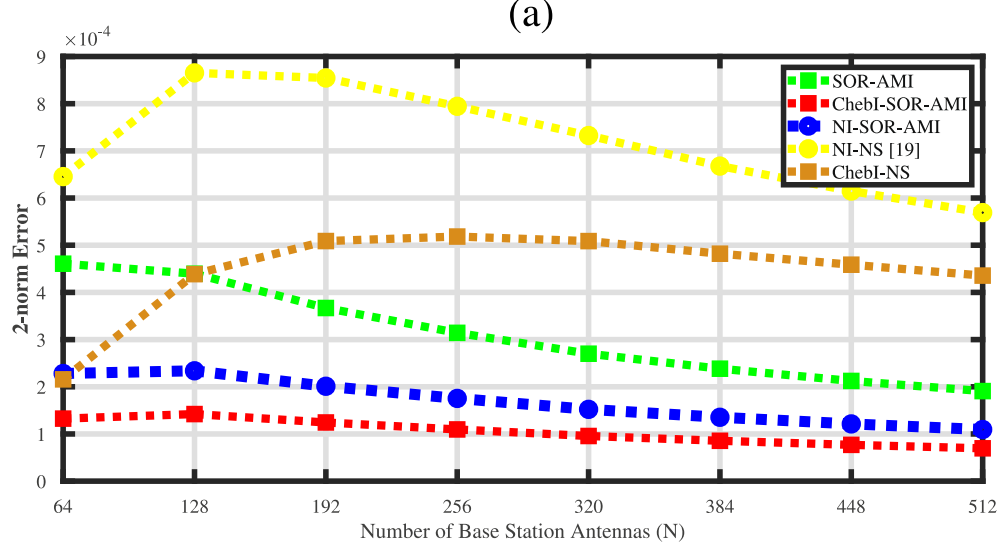

(b)

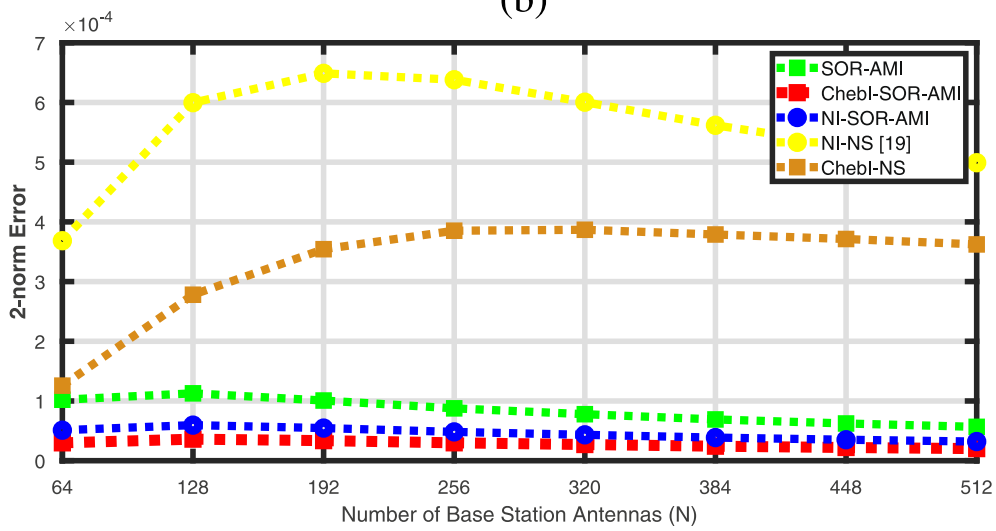

(c)

Fig. 2 MSE of ChebI-NS, NI-NS, SOR-AMI, ChebI-SOR-AMI, and NI-SOR-AMI methods versus number of BS antennas ( $N$ ) for uncorrelated channel at the second, third, and fourth iterations, respectively. This figure computes the MSE of four approximate matrix inversion techniques for uncorrelated channel conditions under second, third, and fourth iterations, respectively. a iteration, $i=2$. $\mathbf{b}$ iteration, $i=3$. c iteration, $i=4$

due to low utilized iterations. In the third and fourth iteration, i.e., Fig. 3b, c, e, and f, ChebI-NS is the nearest method to optimal performance. Because NS has a slow convergence rate, it requires more than two iterations for more accurate matrix inversion. Therefore at these conditions, the new ChebI-NS method gains a superior performance more than other NS approaches ensuring its fastest convergence.
Figure 4 shows the second and fourth iteration of the BER against SNR of new proposed SOR-AMI, NI-SORAMI, ChebI-SOR-AMI, and SSOR with $N \times K=265 \times$ 32 under uncorrelated channel, Fig. 4 a and b, and correlated channel, Fig. 4c and d, respectively. From the figure, the new Cheb-SOR-AMI algorithm has the superior performance close to ZF followed by the NI-SORAMI method. The results at second iteration, i.e., Fig.4a 


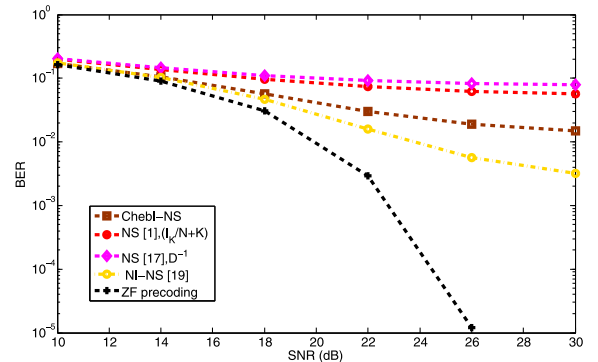

(a)

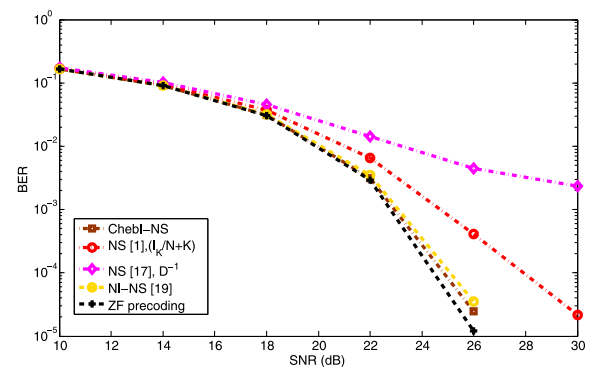

(c)

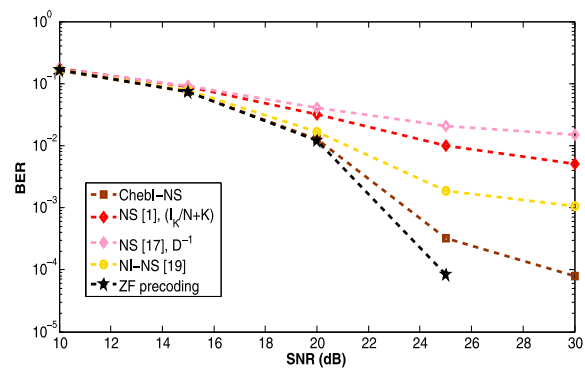

(e)

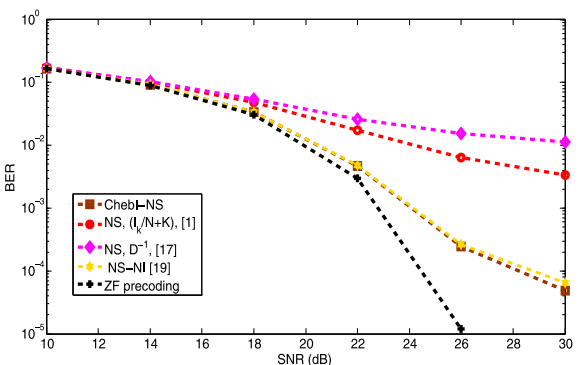

(b)

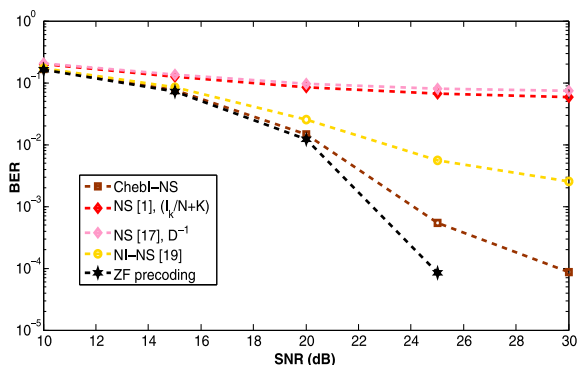

(d)

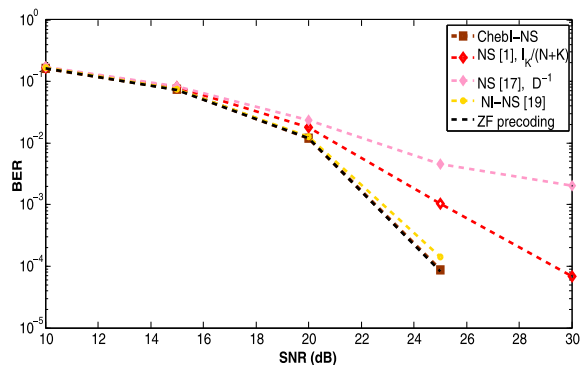

(f)

Fig. 3 BER performance comparison between different joint NS methods with Chebl-NS method in mMIMO system with $N=128$ and $K=16$ under uncorrelated and correlated channel, respectively. This figure compares Chebl-NS with other previous NS methods for iterations $i=2,3,4$ under both correlated and uncorrelated channel conditions. a Uncorrelated channel, $i=2$. b Uncorrelated channel, $i=3$. c Uncorrelated channel, $i=4$. d Correlated channel, $i=2$. e Correlated channel, $i=3$. $\mathbf{f}$ Correlated channel, $i=4$

and c, indicate that both ChebI-SOR-AMI and NI-SORAMI converge fast to the optimal performance; however, at the fourth iteration, all methods have close performance to ZF.

Figure 5 presents a performance comparison among the new proposed methods under uncorrelated channels, Fig. $5 \mathrm{a}$ and $\mathrm{b}$, and correlated channels, Fig. $5 \mathrm{c}$ and $\mathrm{d}$, for the second and fourth iterations, with $N=128$ and $K=16$, respectively. In correlated channel results, the BER error floor for the proposed methods show a good convergent trend similar to uncorrelated channels. ChebI-SOR-AMI has much better performance than other methods. SORAMI convergence speed is faster than NS; hence, SORAMI-based methods provide accurate results at lower iterations. Also, at low iterations, we recommend utilizing NI-SOR-AMI than ChebI-SOR-AMI as it has close result with reduced complexity. ChebI-NS has low performance due to the slower convergence of NS than SOR method. However, ChebI-NS is preferable for designers due to the ease of NS hardware implementation. Also, our proposed methods are robust to channel correlation more than existing NS, and SOR methods.

\section{Conclusion}

In this paper, we have investigated the slow convergence of both NS and SOR methods in linear precoding. For this purpose, we have proposed four joint methods to calculate ZF linear precoding weights for mMIMO systems, i.e., ChebI-NS, SOR-AMI, NI-SOR-AMI, and ChebI-SOR-AMI. ChebI-NS has been proposed to speed up the convergence of NS and also to give more accurate approximation. Unlike traditional SOR method, 


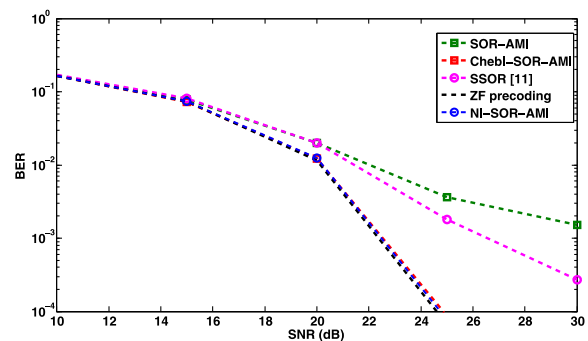

(a)

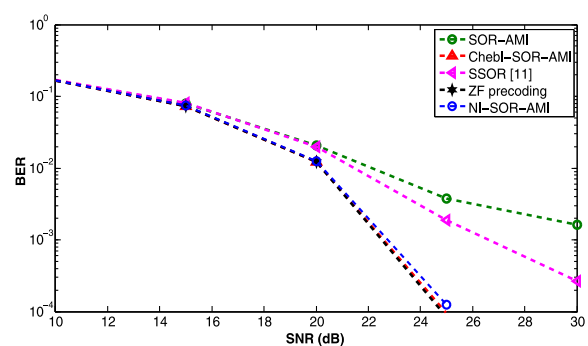

(c)

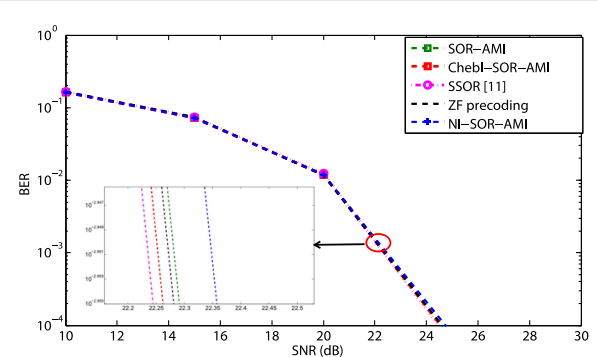

(b)

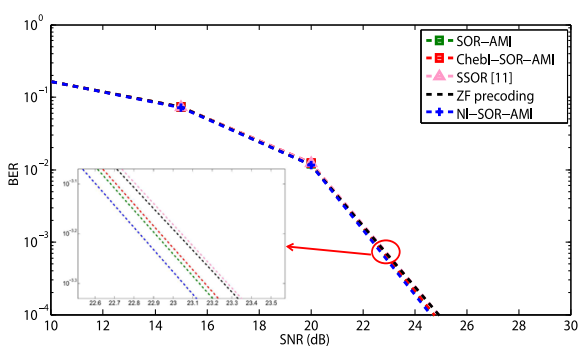

(d)

Fig. 4 BER performance comparison between SSOR, NI-SOR-AMI, and Chebl-SOR-AMI method $N \times K=256 \times 32$ mMIMO system for uncorrelated and correlated channel conditions. This figure compares SOR-AMI, Chebl-SOR-AMI, and NI-SOR-AMI with conventional SOR and SSOR methods under correlated and uncorrelated channel conditions for the second and fourth iterations. a Uncorrelated channel, $i=2$. b Uncorrelated channel, $i=4$. c Correlated channel, $i=2$. d Correlated channel, $i=4$

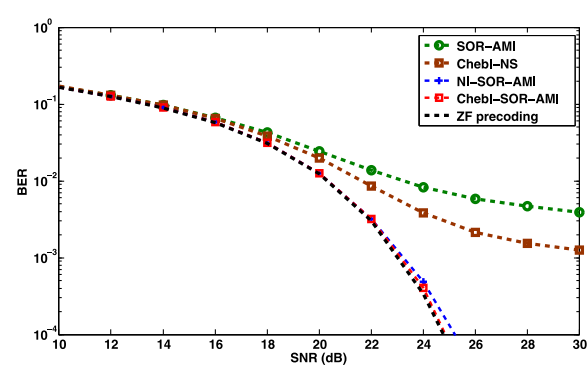

(a)

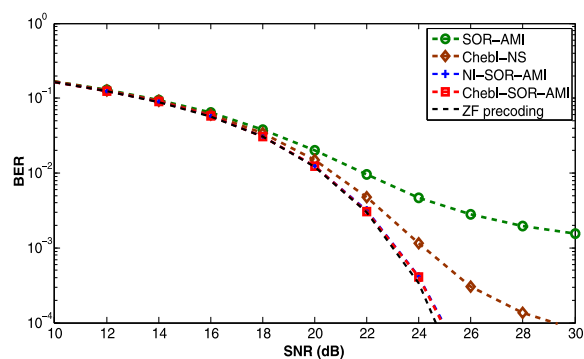

(c)

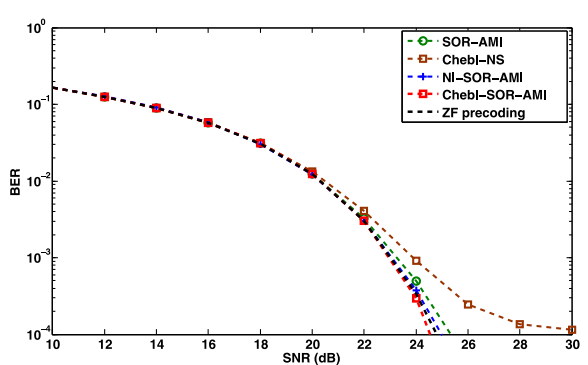

(b)

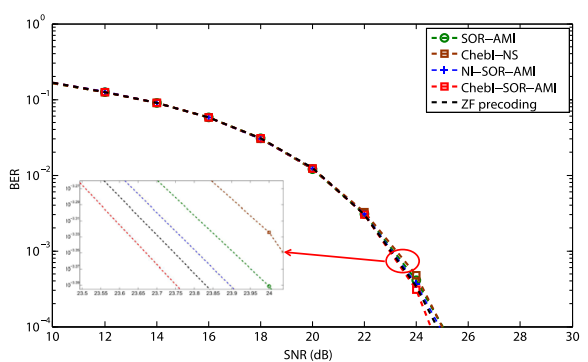

(d)

Fig. 5 BER performance comparison between the new proposed methods with $N \times K=128 \times 16 \mathrm{mMIMO}$ system for uncorrelated and correlated channel conditions. This figure compares between the proposed methods in this paper under correlated and uncorrelated channel conditions for the second and fourth iterations respectively. a Uncorrelated channel, $i=2$. b Uncorrelated channel, $i=4$. c Correlated channel, $i=2$. $\mathbf{d}$ Correlated channel, $i=4$ 
SOR-AMI method directly calculates the matrix inversion without multiplying the symbol vector. Joint ChebISOR-AMI and NI-SOR-AMI are based on applying fast converging Chebyshev/Newton iteration as initial step of SOR-AMI method. Simulation results illustrate that the proposed methods give not only accurate results but also fast convergence under both uncorrelated and correlated channel conditions. ChebI-NS method is the fastest among NS-based methods. NI-SOR-AMI is more preferable than ChebI-SOR-AMI as it achieves close performance to ChebI-SOR-AMI with lower complexity. Although NS convergence is lower than SOR-AMI, it is preferable in hardware implementation. Hence, ChebI-NS is important to accelerate the convergence of such a prominent method. Further investigations of the proposed methods with other linear precoders like MMSE and RZF is under consideration as future work.

\section{Appendix A Proof of Lemma 1}

First, we will approve the convergence of ChebI-NS, i.e., $\sum_{n=0}^{\infty}\left(I_{K}-\mathbf{Z}^{(1)} \mathbf{W}\right)^{n} \mathbf{Z}^{(1)}$ then from the convergence approval, the theory is proofed.

According to Eq. (6), the condition of convergence of ChebI-NS is $\lim _{n \rightarrow \infty}\left(\mathbf{I}_{K}-\mathbf{Z}^{(1)} \mathbf{W}\right)^{n}=\mathbf{0}_{K}$.

Let matrix $\mathbf{A}=\mathbf{I}_{K}-\mathbf{Z}^{(1)} \mathbf{W}$.

Since, $\rho\left(\mathbf{I}_{K}-\mathbf{Z}^{(1)} \mathbf{W}\right)<1 \leftrightarrow\left|\lambda_{i}(A)\right|<1$,

where $\lambda_{i}(\mathbf{A})$ denotes the $i^{t h}$ eigenvalue of $\mathbf{A}, 1 \leqslant i \leqslant \mathrm{k}$, $\rho\left(\mathbf{I}_{K}-\mathbf{Z}^{(1)} \mathbf{W}\right)=\rho(\mathbf{A})<1$,

where $\rho(\mathbf{A})$ is the spectral radius of $\mathbf{A}$ i.e., $\lambda_{\max }(\mathbf{A})$ the largest absolute value of $\mathbf{A}$ eigenvalues

Substituting the value of $\mathbf{Z}^{(1)}$ from Eq. (16) into the matrix A yields

$$
\begin{aligned}
\mathbf{A} & =\mathbf{I}_{K}-\mathbf{Z}^{(1)} \mathbf{W}=\mathbf{I}_{K}-\mathbf{D}^{-1}\left(3 \mathbf{I}-\mathbf{W} \mathbf{D}^{-1}\left(3 \mathbf{I}-\mathbf{W} \mathbf{D}^{-1}\right)\right) \mathbf{W} \\
& =\left(\mathbf{I}_{K}-\mathbf{D}^{-1} \mathbf{W}\right)^{3} .
\end{aligned}
$$

Let $\mathbf{B}=\mathbf{I}_{K}-\mathbf{D}^{-1} \mathbf{W}$ then $\sum_{n=0}^{\infty}\left(\mathbf{I}_{K}-\mathbf{D}^{-1} \mathbf{W}\right)^{n} \mathbf{D}^{-1}$ converges to $\left|\lambda_{i}(\mathbf{B})\right|<1$ which have a high probability convergence as in Lemma 1 [16].

Since, $\mathbf{A}=\mathbf{B}^{3}$ and $\lambda_{i}(\mathbf{A})=\lambda_{i}(\mathbf{B})^{3}$

then $\quad \sum_{n=0}^{\infty}\left(\mathbf{I}_{K}-\mathbf{Z}^{(1)} \mathbf{W}\right)^{n} \mathbf{Z}^{(1)} \quad$ converges as $\leftrightarrow \sum_{n=0}^{\infty}\left(\mathbf{I}_{K}-\mathbf{D}^{-1} \mathbf{W}\right)^{n} \mathbf{D}^{-1}$ converges too.

This approves the convergence of ChebI-NS.

From equations (11- 17) in [16], a high probability convergence condition for

$\sum_{n=0}^{\infty}\left(\mathbf{I}_{K}-\mathbf{D}^{-1} \mathbf{W}\right)^{n} \mathbf{D}^{-1}$ equals $\eta>\frac{1}{(\sqrt{2}-1)^{2}}$, i.e., $\eta>$ 5.83. Thus, since we approved the convergence of ChebINS, the same high probability convergence for ChebI-NS is achieved when $\eta>5.83$ [16]. Hence, the proof is finished.

\section{Appendix B Proof of Lemma 2}

Substituting $\mathbf{R}^{(0)}=\mathbf{D}^{-1}$ into $\mathbf{g}^{(0)}=\mathbf{D}^{-1} \mathbf{s}$ yields the following:

$$
\mathbf{g}^{(0)}=\mathbf{R}^{(0)} \mathbf{s}
$$

Hence, for $k_{t h}$ iteration, $\mathbf{g}^{(k)}=\mathbf{R}^{(k)} \mathbf{s}$. Substituting this result in SOR method i.e. Eq. (10), $\mathbf{R}^{(k+1)}$ is obtained as

$$
\begin{aligned}
\mathbf{g}^{(k+1)} & =(\mathbf{D}+\alpha \mathbf{L})^{-1}\left(\alpha \mathbf{s}+((1-\alpha) \mathbf{D}-\alpha \mathbf{U}) \mathbf{g}^{(k)}\right) \\
& =(\mathbf{D}+\alpha \mathbf{L})^{-1}\left(\alpha \mathbf{s}+((1-\alpha) \mathbf{D}-\alpha \mathbf{U}) \mathbf{R}^{(k)} \mathbf{s}\right)=\mathbf{R}^{(k+1)} \mathbf{s}
\end{aligned}
$$

Hence, based on the mathematical induction, we can obtain the following:

$$
\mathbf{g}^{(n)}=\mathbf{R}^{(n)} \mathbf{s},(n \geq 0) .
$$

Equation (26) ends the proof.

\section{Abbreviations}

5G: 5th generation; BER: Bit error rate; BS: Base station; CE: Constant enveloper; CG: Conjugate gradient; ChebI-NS: Joint Chebyshev and NS method; Chebl-SOR-AMI: Joint Chebyshev and SOR-based approximate matrix inversion; Chebl: Chebyshev iteration; CSE: Channel state information; DL: Down link; DPC: Dirty paper coding; GS: Gauss Siedel; i.i.d: Independent and identically distributed; MF: Matched filter; mMIMO: Massive MIMO; MMSE: Minimum mean square error; MSE: Mean square error; NI-Ns: Joint Newton iteration and NS method; NI-SOR-AMI: Joint Newton iteration and SOR-based approximate matrix inversion; NI: Newton iteration; NS: Neumann series; PZF: Phased zero forcing; QAM: Quadrature amplitude modulation; RV: Random variable; RZF: Regularized zero forcing; SNR: Signal to noise ratio; SOR-AMI: SOR-based approximate matrix inversion; SOR: Successive over relaxation; SSOR: Symmetric successive over relaxation; TDD: Time division duplex; THP: Tomlinson Harashima precoding; VP: Vector perturbation; ZF: Zero forcing

\section{Acknowledgements}

Our sincere thanks to MOHE and Center for Japan-Egypt Cooperation in Science and Technology, Kyushu University, Japan, for their guidance, support and encouragement.

\section{Authors' contributions}

Both authors contributed to the design and analysis of the research, to the simulation results, and to the writing of the manuscript. They also discussed the results and contributed to the final manuscript. Both authors read and approved the final manuscript.

\section{Authors' information}

Sherief Hashima was born in El-Santa, Gharbiya, Egypt in 1983. He received his B.Sc. and M.Sc. degrees in Electronics and Communication Engineering (ECE), with class of honors, in 2004, 2010 from Tanta and Menoufiya University, respectively. He obtained his Ph.D degree from Egypt-Japan University of Science \& Technology (EJUST), Alexandria, EGYPT at 2014. He is working as assistant professor at the Engineering and scientific equipment Department, Nuclear Research Center (NRC), Egyptian Atomic Energy Authority (EAEA), Egypt since 2014. From Jan-June 2018, he was a visiting researcher at Center for Japan-Egypt Cooperation in Science and Technology, Kyushu University. Recently, he joined computational learning theory team, RIKEN AIP, Japan as a postdoctoral researcher since July 2019. His research interests include wireless communications, machine learning, online learning, 5G systems, image processing, nuclear instruments design, and internet of things.

Osamu Muta received a B.E. degree from Ehime University, Ehime, Japan, in 1996, an M.E., and Ph.D. degrees from Kyushu University, Fukuoka, Japan in 1998, and 2001 respectively. In 2001, he joined the Graduate School of Information Science and Electrical Engineering, Kyushu University as an assistant professor. Since 2010, he has been an associate professor in Center for Japan-Egypt Cooperation in Science and Technology, Kyushu University. 
His research interests include signal transmission processing techniques for high-speed wireless communications and power-line communications, and non linear distortion compensation techniques for high-power amplifiers. He received the 2005 Active Research Award for excellent presentation from IEICE Radio Communication Systems. Dr. Muta is a senior member of IEICE and a member of IEEE.

\section{Funding}

This research was supported in part by the JSPS KAKENHI (JP17K06427) and in part by the Egyptian Ministry of Higher Education (MoHE).

\section{Competing interests}

The authors declare that they have no competing interests.

\section{Author details}

${ }^{1}$ Engineering Dept, Egyptian Atomic Energy Authority, 13759 Inshas, Egypt. ${ }^{2}$ Center for Japan-Egypt Cooperation in Science and Technology, Kyushu University, 819-0395 Fukuoka, Japan.

Received: 23 February 2019 Accepted: 18 December 2019 Published online: 04 February 2020

\section{References}

1. F. Rusek, et al., Scaling up MIMO: opportunities and challenges with very large arrays. IEEE Signal Process. 30(1), 40-60 (2013). https://doi.org/10. 1109/MSP.2011.2178495

2. T. Marzetta, E. Larsson, H. Yang, H. Ngo, Fundamentals of Massive MIMO. (Cambridge University Press, 2016). https://doi.org/10.1017/ CBO9781316799895

3. E. Bjrnson, J. Hoydis, L. Sanguinetti, Massive MIMO networks: spectral, energy, and hardware efficiency. Found. Trends Signal Process. (2018). http://dx.doi.org/10.1561/2000000093

4. M. Costa, Writing on dirty paper (Corresp.) IEEE Trans. Informa. Theory. 29(3), 439-441 (1983)

5. M. Mazrouei-Sebdani, W. A. KrzymieÅ, J. Melzer, Massive MIMO with nonlinear precoding: large-system analysis. IEEE Trans. Veh. Technol. 65(4), 2815-2820 (2016). https://doi.org/10.1109/TVT.2015.2425884

6. N. Fatema, G. Hua, Y. Xiang, D. Peng, I. Natgunanathan, Massive MIMO linear precoding: a survey. IEEE Syst. J, 1-12 (2017). https://doi.org/10.1109/ JSYST.2017.2776401

7. ke Bjrck, Numeric Methods in Matrix Computations. (Springer, 2015). https:// doi.org/10.1007/978-3-319-05089-8

8. X. Gao, L. Dai, Y. Ma, Z. Wang, Low-complexity near-optimal signal detection for uplink large-scale MIMO systems. Electron. Lett. 50(18), 1326-1328 (2014). https://doi.org/10.1049/el.2014.0713

9. B. Yin, M. Wu, J. R. Cavallaro, C. Studer, Conjugate gradient-based soft-output detection and precoding in massive MIMO systems. IEEE Glob. Commun. Conf. (GLOBECOM), 3696-3701 (2014). https://doi.org/10.1109/ GLOCOM.2014.7037382

10. T. Xie, Q. Han, H. Xu, Z. Qi, W. Shen, in 81st IEEE Vehicular Technology Conference. A low-complexity linear precoding scheme based on SOR method for massive MIMO systems (VTC Spring, 2015), pp. 1-5. https://doi. org/10.1109/NTCSpring.2015.7145618

11. T. Xie, L. Dai, X. Gao, X. Dai, Y. Zhao, Low-complexity SSOR-based precoding for massive MIMO systems. IEEE Commun. Lett. 20(4), 744-747 (2016). https://doi.org/10.1109/LCOMM.2016.2525807

12. X. Gao, L. Dai, J. Zhang, S. Han, I. Chih-Lin, Capacity-approaching linear precoding with low-complexity for large-scale MIMO systems. IEEE Int. Conf. Commun. (ICC), 1577-1582 (2015). https://doi.org/10.1109/ICC.2015. 7248549

13. L. Shao, Y. Zu, Approaches of approximating matrix inversion for zero-forcing precoding in downlink massive MIMO systems. Springer J. Wirel. Netw. (2017). https://doi.org/10.1007/s11276-017-1496-z

14. C. Tang, C. Liu, L. Yuan, Z. Xing, High precision low complexity matrix inversion based on Newton iteration for data detection in the massive MIMO. IEEE Commun. Lett. 20(3), 490-493 (2016). https://doi.org/10.1109/ LCOMM.2015.2514281

15. C. Zhang, Z. Li, L. Shen, F. Yan, M. Wu, X. Wang, A low-complexity massive MIMO precoding algorithm based on Chebyshev iteration. IEEE Access. $\mathbf{5}$, 22545-22551 (2017). https://doi.org/10.1109/ACCESS.2017.2760881
16. D. Zhu, B. Li, P. Liang, On the matrix inversion approximation based on neumann series in massive MIMO systems. IEEE Int. Conf. Commun. (ICC), 1763-1769 (2015). https://doi.org/10.1109/ICC.2015.7248580

17. H. Prabhu, J. Rodrigues, O. Edfors, F. Rusek, Approximative matrix inverse computations for very-large MIMO and applications to linear pre-coding systems. IEEE Wirel. Commun. Netw. Conf. (WCNC), 2710-2715 (2013). https://doi.org/10.1109/WCNC.2013.6554990

18. A. Thanos, Algorithms and Hardware Architectures for Matrix Inversion in Massive MIMO Uplink Data Detection, M.Sc. Thesis. (University of PATRAS, 2017)

19. L. Shao, Y. Zu, Joint Newton iteration and Neumann series method of convergence accelerating matrix inversion approximation in linear precoding for massive MIMO systems. Hindawi J. Math. Probl. Eng. (2016). http://dx.doi.org/10.1155/2016/1745808

20. M. Wu, B. Yin, G. Wang, C. Dick, J. R. Cavallaro, C. Studer, Large-Scale MIMO Detection for 3GPP LTE: Algorithms and FPGA Implementations. IEEE J. Sel. Top. Signal Process. 8(5), 916-929 (2014). https://doi.org/10.1109/JSTSP. 2014.2313021

21. F. Rosrio, F. A. Monteiro, A. Rodrigues, Fast matrix inversion updates for massive MIMO detection and precoding. IEEE Signal Process. Lett. 23(1), 75-79 (2016). https://doi.org/10.1109/LSP.2015.2500682

22. L. Lu, G. li, A. Swindlehurst, A. Ashikhmin, R. Zhang, An overview of massive MIMO: benefits and challenges. IEEE J. Sel. Top. Signal Process. 8(5), 742-758 (2014). http://doi.org/10.1109/JSTSP.2014.2317671

23. T. L. Marzetta, Noncooperative cellular wireless with unlimited numbers of base station antennas. IEEE Trans. Wirel. Commun. 9(11), 3590-3600 (2010). http://doi.org/10.1109/TWC.2010.092810.091092

24. L. Dai, X. Gao, X. Su, S. Han, C.L. I., Z. Wang, Low-complexity soft-output signal detection based on Gauss-Seidel method for uplink multiuser large-scale MIMO systems. IEEE Trans. Veh. Technol. 64(10), 4839-4845 (2015). http://doi.org/10.1109/TVT.2014.2370106

25. E. Godana, T. Ekman, Parametrization based limited feedback design for correlated MIMO channels using new statistical models. IEEE Trans. Wirel. Commun. 12(10), 5172-5184 (2013). http://doi.org/10.1109/TWC.2013. 092013.130045

26. C. He, R. D. Gitlin, Limiting performance of massive MIMO downlink cellular systems. Informa. Theory Appl. Workshop. ITA, 1-6 (2016). http:// doi.org/10.1109/ITA.2016.7888139

\section{Publisher's Note}

Springer Nature remains neutral with regard to jurisdictional claims in published maps and institutional affiliations.

\section{Submit your manuscript to a SpringerOpen ${ }^{\circ}$ journal and benefit from:}

- Convenient online submission

- Rigorous peer review

- Open access: articles freely available online

- High visibility within the field

- Retaining the copyright to your article

Submit your next manuscript at $\gg$ springeropen.com 